\title{
Patient effective dose estimation for routine computed tomography examinations in Iran
}

\author{
M.R. Deevband ${ }^{*}$, M. Ghorbani ${ }^{1}$, A. Eshraghi ${ }^{2}$, Y. Salimi ${ }^{1}$, \\ E. Saeedzadeh ${ }^{2}$, M.R. Kardan3, S. Sadeghi4, D. Divband4, M. Ahmadi 1 \\ ${ }^{1}$ Biomedical Engineering and Medical Physics Department, School of Medicine, Shahid Beheshti University of \\ Medical Sciences, Tehran, Iran. \\ ${ }^{2}$ Department of Medical Radiation Engineering, Science and Research Branch, Islamic Azad \\ University, Tehran, Iran \\ ${ }^{3}$ Nuclear Sciences Research School, Nuclear Sciences and Technology Research Institute, Tehran, Iran \\ ${ }^{4}$ Scool of Medicine, Islamic Azad University, Tehran, Iran
}

\section{- Original article}

\section{*Corresponding authors: \\ Mohammad R. Deevband, PhD., E-mail:}

Mdeevband@sbmu.ac.ir

Revised: January 2020

Accepted: February 2020

Int. J. Radiat. Res., January 2021; 19(1): 63-73

DOI: $10.29252 /$ ijrr.19.1.63

\begin{abstract}
Background: The present study intended to determine and report patient effective dose on the basis of patients and exposure data. Materials and methods: A nationwide computed tomography (CT) survey was provided as a report of patient doses in 2015-2016. Scan details were collected for nearly 2,000 adults and children in four age groups subjected to CT examinations. From total 565 CT scanners in different models in Iran, 120 different scanners were sampled. ImpactDose software was used to calculate the effective dose (ED) by collecting the necessary data also as an alternative fast method, the ED was estimated by multiplying dose length product (DLP) and a conversion factor. Results: There was a high variation in doses received by patients. The estimated EDs by the DLP and conversion factor were lower (except for sinus protocol) than those by ImpactDose software $(p=0.014)$. The mean EDs were $1.09,0.66,7.70$ and $13.29 \mathrm{mSv}$ for adult patients' procedures of head, sinus, chest and abdomen-pelvis, respectively. In terms of CTDIvol and DLP, in Iran the mean effective doses were significantly lower than other countries. Conclusion: Publishing guidelines and exposure tables according to patient situations is necessary to decrease variations in doses and exposure parameters. Since the DLP conversion factor leads to a considerable discrepancy in calculating ED, when there is a need for precise dose calculations, the DLP conversion factor should not be used. Furthermore, it is suggested that ED be used as DRL, instead of CTDIvol.
\end{abstract}

Keywords: Effective dose estimation, CTDI, DLP, impact dose software.

\section{INTRODUCTION}

Computed tomography (CT) capacity for providing high-quality three-dimensional data has significant benefits for the medical management as it provides fast and accurate diagnosis and prevents surgical techniques $(1,2)$. However, the CT is accompanied by relatively high radiation doses to patients; hence, it has higher risk of carcinogenesis (3-5). The sensible use of this modality is applied by the adequate performance of radiation protection, justification, optimization and minimization to ensure a low risk to patients and simultaneously have benefits due to this technique (6).

The first step to reach optimization rule is collecting information about received radiation doses by patients in order to develop diagnostic reference levels (DRLs) (7). Despite the lack of dose limit to medical exposure, high dose levels can be identified by establishment of DRL to allow benefits of the radiation. Therefore, the 
knowledge about typical dose levels enables CT users to identify and address those protocols which are not according to as low as reasonably achievable (ALARA) principle.

The clinical benefits of a CT imaging technique for healthcare were immediately recognized after its application in clinical practices in $1973{ }^{(8)}$. The use of CT has increased until now (9-12). Due to this increasing trend, the contribution of CT is increasing from a total collective dose $(13,14)$.

The application of CT has increased in clinical practice due to the development of CT technology because of the introduction of multislice scanners and obtaining lower scan time (15). The International Commission on Radiological Protection (ICRP) recommends that DRLs are based on the relevant local, regional or national data (16). It is thus necessary to establish the Iranian DRLs to ensure the optimization in Iran.

A number of studies have been conducted on the determination of DRL in different cities of Iran (17-19). The national DRL was also determined in a study by Sohrabi, et al. (19). However, in the study by Sohrabi, et al., the DRL was not determined for children, and also different methods were not compared for the DRL specification. The aim of the present study is to investigate the radiation effective doses and to establish national DRLs, based on the ICRP 103 report (20), for pediatrics and adults groups in CT examinations for Iran. Determination of DRL for pediatric and adult groups by using different methods are new aspects of the present study.

\section{MATERIALS AND METHODS}

In the present study the radiation doses in pediatric and adult underwent CT examinations in Iran was evaluated. Based on the data, national DRLs were established for the most common CT examinations using the dose length product (DLP), and CT volume dose index (CTDIvol) of primary dosimetry metrics. In addition, effective dose and DRL was calculated to yield a quantity to estimate the radiation risk comparable among different CT protocols.

\section{Calibration of dose indicators}

A CT dosimetry set including a pencil-shaped ionization chamber (DCT10 RS, RTI Electronics, Sweden), a Barracuda electrometer with active length of $10 \mathrm{~cm}$, and two CT dosimetry phantoms were utilized for measurement of CTDIvol values. The phantoms had constructed cylindrical shape from polymethylmethacrylate (PMMA) with diameters of $16 \mathrm{~cm}$ and $32 \mathrm{~cm}$ and length of $15 \mathrm{~cm}$ for head and body measurements, respectively. Calibration of the ionizing chamber was performed in Secondary Standard Dosimetry Laboratory (SSDL, Karaj, Iran). The cylindrical PMMA phantoms were fixed in the isocenter of gantry of CTs. Measurements were performed according to the guidelines of International Electrotechnical Commission (IEC, publication no. 60601-2-44). The phantoms were scanned by different routine $\mathrm{kVps}$ and $100 \mathrm{mAs}$ fixed tube current. The reported CTDIvol and DLP values by CT consoles were compared with the measured data. The conversion factor for each $\mathrm{kVp}$ was calculated by dividing the measurement value by the CT reported value. These conversion factors were implemented for each center to yield the real CTDIvol, and DLP.

\section{CT scanners and patients characteristics}

In table 1 the details of survey based on scanner models is presented. During the period of this study, from total 565 different models of CT scanners in in Iran, 120 different scanners were sampled. In general, $45 \%$ out of the 120 sampled scanners were Siemens, 25\% General Electric (GE), 10\% Toshiba, 10\% Neusoft, 10\% Shimadzu and 1\% Philips. According to large number of sampling, the sampled scanners were representative of the total CT scanners throughout Iran. It is also evident from this table that, the most frequent CT scanner, both in Iran and in the sample, is Siemens unit. The percentage of most frequent CT examinations in Iran during one month is presented in table 2 . Based on this table, the most frequent CT examinations in Iran are the head CT examinations. Table 3 present the CT 
examination parameters for different age groups in Iran. It is found that the most frequent examination is related to the head protocol (12).

The data was collected for four procedures on the head (brain), sinus, chest and abdomen-pelvis. Questionnaires were collected from covering CT scanners data, patients' data and scan parameters. Scanner data included names, models, numbers of detector rows, and conversion factor for dose. The collected scanning data included the peak tube potential, tube current, tube current modulation (TCM) systems, slice thickness, pitch and scan range. All of CT systems were equipped with TCM systems. The survey was begun in 2015 and completed at the end of 2016. During a two-year period, 120 questionnaires were returned to the Biomedical Engineering and Medical Physics Department of Shahid Beheshti University of Medical Sciences as separate data submissions from 120 scanners located at 106 hospitals of Iran.

Table 1. The CT scanner models in Iran and numbers of samples for each scanner model, during the period of this study.

\begin{tabular}{|c|c|c|c|c|c|c|c|c|}
\hline & Siemens & General electric & Toshiba & Neusoft & Shimadzu & Philips & Hitachi & Picker \\
\hline Number of systems in Iran & 225 & 142 & 60 & 64 & 47 & 16 & 7 & 4 \\
\hline Number of systems in the sample & 54 & 25 & 18 & 10 & 9 & 1 & 0 & 0 \\
\hline
\end{tabular}

Table 2. Percentage (\%) of most frequent CT examinations in Iran during one month.

\begin{tabular}{|c|c|c|c|c|c|}
\hline Chest & Abdomen & Pelvic & Head & More than one organ & Others \\
\hline 8 & 10 & 9 & 57 & 7 & 9 \\
\hline
\end{tabular}

Table 3. Analysis of applied settings in pediatrics (up to 1 year old, 1-5, 6-10, and 11-15 years old) and adult standard examination protocols (brain, sinus, chest, and abdomen-pelvis).

\begin{tabular}{|c|c|c|c|c|c|c|c|c|c|}
\hline Age range & Examination & Phases & Sequences & KVp & mAs & Pitch & $\begin{array}{c}\text { Slice thickness } \\
\text { (mm) }\end{array}$ & $\begin{array}{l}\text { No. of } \\
\text { slices }\end{array}$ & $\begin{array}{c}\text { Scan length } \\
\text { (cm) }\end{array}$ \\
\hline \multirow{4}{*}{$\begin{array}{c}\text { Up to } 1 \text { year } \\
\text { old }\end{array}$} & Brain & 1 & 1 & $110-130$ & $50-180$ & $0.4-0.8$ & $4-8$ & $7-23$ & - \\
\hline & Sinus & 1 & 1 & $100-130$ & $30-60$ & $0.5-0.8$ & $3.75-5$ & $11-15$ & - \\
\hline & Chest & 1 & 1 & $100-130$ & $50-130$ & $0.8-1.15$ & $5-10$ & $12-24$ & - \\
\hline & Abdomen-pelvis & 1 & 1 & $110-130$ & $70-180$ & $0.8-1.37$ & $5-10$ & $14-36$ & - \\
\hline \multirow{4}{*}{$\begin{array}{l}\text { Ages ranging } \\
\text { from } 1 \text { to } 5\end{array}$} & Brain & 1 & 1 & $110-130$ & $50-180$ & $0.8-1$ & $4-10$ & $8-22$ & - \\
\hline & Sinus & 1 & 1 & $100-130$ & $35-60$ & $0.56-1$ & $3.75-5$ & $11-22$ & - \\
\hline & Chest & 1 & 1 & $100-130$ & $80-130$ & $0.8-1.37$ & $5-10$ & $15-30$ & - \\
\hline & Abdomen-pelvis & 1 & 1 & $110-130$ & $80-200$ & $0.8-1.37$ & $5-10$ & $26-48$ & - \\
\hline \multirow{5}{*}{$\begin{array}{l}\text { Ages ranging } \\
\text { from } 6 \text { to } 10\end{array}$} & \multirow{2}{*}{ Brain } & \multirow{2}{*}{2} & 1 & $110-130$ & $50-180$ & 0.4-1 & $5-10$ & $6-16$ & - \\
\hline & & & 2 & $110-130$ & $50-180$ & $0.4-1$ & 4-10 & $7-16$ & - \\
\hline & Sinus & 1 & 1 & $110-130$ & $35-75$ & $0.56-1$ & $3.75-10$ & $12-28$ & - \\
\hline & Chest & 1 & 1 & $110-130$ & $50-150$ & $0.8-1.37$ & $5-10$ & $16-41$ & - \\
\hline & Abdomen-pelvis & 1 & 1 & $100-130$ & $80-150$ & $0.8-1.35$ & $5-10$ & $21-68$ & - \\
\hline \multirow{4}{*}{$\begin{array}{l}\text { Ages ranging } \\
\text { from } 11 \text { to } 15\end{array}$} & Brain & & & $110-130$ & $70-300$ & $0.8-1.2$ & $4-10$ & $4-18$ & $5.5-35.5$ \\
\hline & Sinus & & & $110-130$ & $30-290$ & $0.8-1.5$ & $3.75-5$ & $12-30$ & $6.6-22$ \\
\hline & \begin{tabular}{|c|} 
Chest \\
\end{tabular} & & & $110-140$ & $45-290$ & 1-1.4 & $5-10$ & $20-40$ & $13.5-62.8$ \\
\hline & Abdomen-pelvis & & & $100-130$ & $70-300$ & $1-1.4$ & $5-10$ & $22-80$ & $30.6-71.3$ \\
\hline \multirow{5}{*}{ Adults } & \multirow{2}{*}{ Brain } & \multirow{2}{*}{2} & 1 & 110-130 & $100-300$ & 0.8- 1.5 & $5-10$ & $5-18$ & 5.9- 36.1 \\
\hline & & & 2 & $110-130$ & $70-290$ & $0.8-1.5$ & $4-10$ & $6-18$ & $6.8-23.3$ \\
\hline & Sinus & 1 & 1 & $100-130$ & $35-280$ & $0.8-1.5$ & $3.75-5$ & $13-31$ & $6.8-23.3$ \\
\hline & Chest & 1 & 1 & $110-140$ & $50-290$ & $1-1.35$ & $5-10$ & $21-59$ & $13.5-62.8$ \\
\hline & Abdomen-pelvis & 1 & 1 & $110-130$ & $70-300$ & $1-1.35$ & $5-10$ & $24-87$ & $30.6-71.3$ \\
\hline
\end{tabular}


Nevertheless, the present study included a substantial sample (about 21\%) of 565 CT scanners in Iran based on an adequate geographical and technological spread. Detailed information was collected from 1160 patients for each protocol. In the present study, 22 out of 31 provinces ( $82 \%$ of Iran's area) were included in order to define a national diagnostic reference level (NDRL) and appropriately cover the whole country.

The patients' age, gender and size were recorded in terms of lateral and anterior-posterior (AP) diameters. Lateral and AP diameters on axial CT images were used to calculate an effective diameter according to the report number 204 by the American Association of Physicists in Medicine (AAPM) (21). The data of at least 10 patients was recorded for each CT exam.

\section{Effective dose estimation}

The total body and organ effective doses (EDs) were calculated by entering the patient data and acquisition parameters in ImpactDose software (version 2.3 from GMBH Company, Germany). For examinations by the tube current modulation (TCM) method, this option was selected in ImpactDose software. The patient's range of scans was chosen by defined anatomical markers in the software. The EDs of patients were calculated by considering patient age and gender, and weighting factors determined by ICRP report $103^{(20)}$. The calibrated phantom diameter for each protocol was entered in ImpactDose module $(16 \mathrm{~cm}$ or 32 $\mathrm{cm}$ ).

As an alternative method for each examination, the ED was calculated from the DLP using a region and age specific coefficient $(k)$. For this purpose the equation number (5) was used:

$$
\mathrm{E}(\mathrm{mSv})=\mathrm{k} \times \mathrm{DLP}
$$

Where, $k(\mathrm{mSv} /(\mathrm{mGy} . \mathrm{cm}))$ is the normalized value of effective dose per dose-length product (22). It is a factor which is specified by the scanning region and age (22). Comprehensive datasets of such coefficients were reported for six broad regions and five standard ages for the general use (23) based on Monte Carlo dose calculations for a number of mathematical phantoms (23). Table 4 presents data that is mainly independent of scanner model and operating conditions.

Table 4. Effective dose per dose length product or $\mathrm{k}$ conversion factor ( $\mathrm{mSv} /(\mathrm{mGy} . \mathrm{cm}))$ for different CT examination protocols (head, sinus, chest, abdomen and pelvis) for pediatrics (up to 1 year old, 1-5, 6-10, and 11-15 years old) and adults. These data were adopted from the ICRP 103 report $^{(20)}$.

\begin{tabular}{|c|c|c|}
\hline Protocol & Age & $k$ conversion factor \\
\hline \multirow{5}{*}{ Head } & 0-1 year & 0.0087 \\
\hline & $1-5$ years & 0.0054 \\
\hline & $6-10$ years & 0.0035 \\
\hline & $11-15$ years & 0.0027 \\
\hline & Adult & 0.0019 \\
\hline \multirow{5}{*}{ Sinus } & $0-1$ year & 0.0087 \\
\hline & $1-5$ years & 0.0054 \\
\hline & $6-10$ years & 0.0035 \\
\hline & $11-15$ years & 0.0027 \\
\hline & Adult & 0.0019 \\
\hline \multirow{5}{*}{ Chest } & 0-1 year & 0.0739 \\
\hline & $1-5$ years & 0.0482 \\
\hline & $6-10$ years & 0.0323 \\
\hline & $11-15$ years & 0.0237 \\
\hline & Adult & 0.0146 \\
\hline \multirow{5}{*}{ Abdomen } & 0-1 year & 0.0841 \\
\hline & $1-5$ years & 0.053 \\
\hline & $6-10$ years & 0.0357 \\
\hline & $11-15$ years & 0.0249 \\
\hline & Adult & 0.0153 \\
\hline \multirow{5}{*}{ Pelvis } & 0-1 year & 0.0701 \\
\hline & $1-5$ years & 0.0446 \\
\hline & $6-10$ years & 0.03 \\
\hline & $11-15$ years & 0.0219 \\
\hline & Adult & 0.0129 \\
\hline
\end{tabular}

\section{Statistical analysis}

The International Business Machines (IBM) Statistical Package for Social Sciences (SPSS) software (version 22.0, IBM Corporation, Armonk, New York) was used for data analysis. The Kolmogorov-Smirnov test was used to assess the data distribution normality. The independent $t$-test was used to compare doses from MSCTs and single slice CTs (SSCTs). $P$-values less than 4.49 were considered

Int. J. Radiat. Res., Vol. 19 No. 1, January 2021 
statistically significant. Mean and standard deviation (SD) of dose indicators were also calculated. Histograms of dose quantities were plotted, and the third quartile was considered as the NDRL (24),

\section{RESULTS}

Tables 5 and 6 present statistical parameters, namely mean, SD, 3rd quartiles and maximum doses in terms of CTDIvol (mGy) and DLP (mGy.cm), respectively. These quantities are related to different $\mathrm{CT}$ examination protocols (head, sinus, chest and abdomen-pelvis) and patient age groups, up to 1 year old, 1-5, 6-10, 11-15 years old and adults.

In table 7 the effective doses calculated by ImpactDose software and $\mathrm{k}^{*}$ DLP are listed. The data were related to different CT examination protocols (head, sinus, chest and abdomen-pelvis) and patient ages (pediatrics and adults). The $p$-value for comparison of the effective dose data presented in table 7 was less than 0.01, which indicates a significant difference between these two data sets.

The CTDIvol (mGy) values in the present study compared with recent studies for a number of CT protocols and age groups are presented in table 8. The measured CTDIvol (mGy) values from the present study compared with similar studies on the pediatric and adult CT examinations in other countries are illustrated in figures 1 and 2, respectively. The reported DLP from the present study compared with recent studies on pediatric and adult CT examinations in other countries are presented in figures 3 and 4 , respectively. In these presentations (table 8, figures 1 and 4) the data of the other countries are related to Canada (11), Egypt (25), Portugal (26), Switzerland (27) and Tehran (19). Since there was not any study on determination of DRL values in in CT in Iran, the results of the present study were compared with the data related to Tehran (Iran) city. Additionally, there are some cases in these presentations which do not include any data, since the data were not reported in the other studies in these cases.

Table 5. Statistical parameters calculated for CTDIvol (mGy), for different CT examination protocols (head, sinus, chest and abdomen-pelvis) and patient ages (up to 1 year old, 1-5, 6-10, 11-15 years old, and adults) in the present study.

\begin{tabular}{|c|c|c|c|c|c|c|c|c|}
\hline \multicolumn{9}{|c|}{ CTDI $_{\text {vol }}$ (mGy) } \\
\hline Protocol & Age & Mean & Standard deviation & Minimum & Percentile 25 & Median & Percentile 75 & Maximum \\
\hline \multirow{5}{*}{ Head } & $0-1$ year & 21.36 & 9.41 & 13.8 & 15.9 & 19.2 & 23.4 & 57.1 \\
\hline & $1-5$ years & 25.87 & 12.87 & 13.8 & 16.8 & 20.2 & 30.6 & 60 \\
\hline & 6-10 years & 32.61 & 13.91 & 13.2 & 21.05 & 32.1 & 37.2 & 68 \\
\hline & $11-15$ years & 34.78 & 13.01 & 13.1 & 30.1 & 32.1 & 41.1 & 70 \\
\hline & Adult & 35.87 & 16.79 & 6.73 & 25.8 & 32.3 & 44.12 & 154 \\
\hline \multirow{5}{*}{ Sinus } & $0-1$ year & 7.72 & 6.22 & 3.2 & 4.39 & 5.81 & 8.9 & 33.3 \\
\hline & $1-5$ years & 8.11 & 6.34 & 0.95 & 4.39 & 6.12 & 9.31 & 33.3 \\
\hline & 6-10 years & 8.86 & 6.78 & 3.2 & 5 & 8.26 & 9.35 & 40.1 \\
\hline & $11-15$ years & 9.26 & 5.17 & 3.8 & 8.25 & 8.26 & 8.8 & 42.6 \\
\hline & Adult & 11.17 & 7.98 & 3.8 & 7.15 & 8.3 & 9.35 & 42.88 \\
\hline \multirow{5}{*}{ Chest } & 0-1 year & 5.34 & 3.82 & 1.8 & 3.46 & 4.8 & 6.75 & 23 \\
\hline & $1-5$ years & 5.46 & 4.81 & 1.8 & 3.46 & 3.8 & 4.77 & 23 \\
\hline & $6-10$ years & 5.59 & 4.25 & 2.11 & 3.46 & 4.2 & 5.4 & 23 \\
\hline & $11-15$ years & 7.35 & 4.39 & 2.21 & 5.41 & 6.42 & 8.61 & 23 \\
\hline & Adult & 7.78 & 6.43 & 2.3 & 5.3 & 6.8 & 8.6 & 53.36 \\
\hline \multirow{5}{*}{$\begin{array}{c}\text { Abdomen- } \\
\text { pelvis }\end{array}$} & $0-1$ year & 6.39 & 5.2 & 1.78 & 2.85 & 6.1 & 6.48 & 32 \\
\hline & $1-5$ years & 7.29 & 6.63 & 2.06 & 3 & 6.48 & 8.35 & 32 \\
\hline & $6-10$ years & 8.82 & 5.88 & 2.27 & 4.85 & 8.11 & 10.25 & 32 \\
\hline & $11-15$ years & 9.22 & 5.95 & 2.5 & 4.85 & 8.35 & 9.3 & 33.86 \\
\hline & Adult & 9.94 & 5.38 & 3.8 & 7.01 & 8.78 & 11.05 & 50.6 \\
\hline
\end{tabular}

Int. J. Radiat. Res., Vol. 19 No. 1, January 2021 


\section{Deevband et al. / CT-effective dose assessment}

Table 6. Statistical parameters calculated for DLP (mGy.cm), for different CT examination protocols (head, sinus, chest and abdomen-pelvis) and patient ages (up to 1 year old, 1-5, 5-10, 10-15 years old, and adults) in the present study.

\begin{tabular}{|c|c|c|c|c|c|c|c|c|}
\hline \multicolumn{9}{|c|}{ DLP (mGy.cm) } \\
\hline Protocol & Age & Mean & Standard deviation & Minimum & Percentile 25 & Median & Percentile 75 & Maximum \\
\hline \multirow{5}{*}{ Head } & 0-1 year & 164.91 & 59.34 & 110 & 127 & 152 & 182 & 451 \\
\hline & $1-5$ years & 227.39 & 96.92 & 138 & 159 & 195 & 254 & 563 \\
\hline & 6-10 years & 336.77 & 170.11 & 58.6 & 198 & 401 & 412 & 816 \\
\hline & $11-15$ years & 384.15 & 176.89 & 58.6 & 226 & 417 & 471 & 840 \\
\hline & Adult & 521.09 & 267.77 & 58.6 & 376 & 459 & 647 & 1302 \\
\hline \multirow{5}{*}{ Sinus } & $0-1$ year & 52.8 & 40.37 & 20.9 & 32 & 40 & 60 & 225 \\
\hline & $1-5$ years & 59.68 & 42.47 & 24.7 & 36 & 45 & 71 & 241 \\
\hline & $6-10$ years & 78.07 & 54.03 & 31 & 56 & 75 & 85 & 345 \\
\hline & $11-15$ years & 105.66 & 48.38 & 40 & 94 & 100 & 107 & 495 \\
\hline & Adult & 144 & 98.17 & 41.8 & 102 & 114 & 140.8 & 599.4 \\
\hline \multirow{5}{*}{ Chest } & 0-1 year & 69.12 & 57.68 & 27 & 44 & 55.87 & 85 & 368 \\
\hline & $1-5$ years & 83.82 & 76 & 27 & 54 & 56 & 72 & 368 \\
\hline & 6-10 years & 110.43 & 70.33 & 48 & 72 & 72.8 & 117 & 368 \\
\hline & $11-15$ years & 187.55 & 82.62 & 74 & 151 & 171 & 227 & 437 \\
\hline & Adult & 250.27 & 156.59 & 59.43 & 162.2 & 230 & 289 & 1302 \\
\hline \multirow{5}{*}{$\begin{array}{c}\text { Abdomen- } \\
\text { pelvis }\end{array}$} & 0-1 year & 109.32 & 99.92 & 28 & 47 & 107 & 108 & 640 \\
\hline & $1-5$ years & 154.63 & 134.98 & 47 & 61 & 146 & 190 & 640 \\
\hline & $6-10$ years & 262.22 & 115.97 & 78 & 221 & 231 & 302 & 640 \\
\hline & $11-15$ years & 296.08 & 147.59 & 95 & 186 & 261 & 345 & 704 \\
\hline & Adult & 403.23 & 201.54 & 166 & 298.3 & 352.3 & 513.3 & 1504 \\
\hline
\end{tabular}

Table 7. Effective dose (mSv) calculated by Monte Carlo-based ImpactDose software and kxDLP for different CT examination protocols (head, sinus, chest and abdomen-pelvis) and patient ages (up to 1 year old, 1-5, 5-10, 10-15 years old, and adults) in the present study.

\begin{tabular}{|c|c|c|c|c|c|}
\hline Protocol & Age & Mean (ImpactCT) & 3rd quartile (ImpactCT) & Standard Deviation (ImpactCT) & Mean $(k \times D L P)$ \\
\hline \multirow{5}{*}{ Head } & $0-1$ year & 1.81 & 2.00 & 0.65 & 1.39 \\
\hline & $1-5$ years & 1.52 & 1.70 & 0.65 & 1.25 \\
\hline & $6-10$ years & 1.35 & 1.65 & 0.68 & 1.12 \\
\hline & $11-15$ years & 1.61 & 1.98 & 0.74 & 1.68 \\
\hline & Adult & 1.09 & 1.36 & 0.56 & 1.25 \\
\hline \multirow{5}{*}{ Sinus } & $0-1$ year & 1.28 & 1.45 & 0.98 & 2.13 \\
\hline & $1-5$ years & 0.88 & 1.05 & 0.63 & 1.54 \\
\hline & 6-10 years & 0.69 & 0.75 & 0.48 & 1.73 \\
\hline & $11-15$ years & 0.98 & 0.99 & 0.45 & 3.27 \\
\hline & Adult & 0.66 & 0.65 & 0.45 & 2.20 \\
\hline \multirow{5}{*}{ Chest } & 0-1 year & 5.93 & 7.29 & 4.94 & 1.12 \\
\hline & $1-5$ years & 4.79 & 4.11 & 4.34 & 1.17 \\
\hline & 6-10 years & 4.37 & 4.63 & 2.78 & 1.45 \\
\hline & $11-15$ years & 5.36 & 6.49 & 2.36 & 1.73 \\
\hline & Adult & 7.70 & 8.89 & 4.82 & 2.83 \\
\hline \multirow{5}{*}{$\begin{array}{c}\text { Abdomen- } \\
\text { pelvis }\end{array}$} & $0-1$ year & 11.77 & 11.63 & 10.76 & 1.32 \\
\hline & $1-5$ years & 10.20 & 12.53 & 8.90 & 1.18 \\
\hline & $6-10$ years & 11.53 & 13.28 & 5.10 & 1.46 \\
\hline & $11-15$ years & 9.76 & 11.37 & 4.87 & 1.52 \\
\hline & Adult & 13.29 & 16.92 & 6.64 & 2.19 \\
\hline
\end{tabular}


Table 8. CTDIvol values ( $\mathrm{mGy}$ ) in the present study compared with recent studies.

\begin{tabular}{|c|c|c|c|c|c|c|c|c|c|c|c|c|c|}
\hline \multirow[b]{2}{*}{ Age } & \multirow[b]{2}{*}{ Protocol } & \multicolumn{2}{|c|}{ Canada ${ }^{(11)}$} & \multicolumn{2}{|c|}{ Egypt ${ }^{(25)}$} & \multicolumn{2}{|c|}{ Portugal ${ }^{(26)}$} & \multicolumn{2}{|c|}{ Switzerland ${ }^{(27)}$} & \multicolumn{2}{|c|}{ Tehran $^{(19)}$} & \multicolumn{2}{|c|}{ This Study } \\
\hline & & Mean & $\begin{array}{c}\text { Third } \\
\text { quartile }\end{array}$ & Mean & $\begin{array}{c}\text { Third } \\
\text { quartile }\end{array}$ & Mean & $\begin{array}{c}\text { Third } \\
\text { quartile }\end{array}$ & Mean & \begin{tabular}{|c} 
Third \\
quartile
\end{tabular} & Mean & \begin{tabular}{|c|} 
Third \\
quartile
\end{tabular} & Mean & $\begin{array}{c}\text { Third } \\
\text { quartile }\end{array}$ \\
\hline \multirow{4}{*}{$\begin{array}{c}0-1 \\
\text { year }\end{array}$} & Head & 37 & - & - & - & 39.42 & 48.31 & 17 & - & - & - & 21.36 & 23.4 \\
\hline & Sinus & - & - & - & - & - & - & - & - & - & - & 7.72 & 8.9 \\
\hline & Chest & 2.8 & - & - & - & 1.58 & 2.42 & 4.2 & - & - & - & 5.34 & 6.75 \\
\hline & $\begin{array}{l}\text { Abdomen- } \\
\text { pelvis }\end{array}$ & 3.8 & - & - & - & - & - & - & - & - & - & 6.39 & 6.48 \\
\hline \multirow{4}{*}{$\begin{array}{c}1-5 \\
\text { years }\end{array}$} & Head & 49 & - & - & - & 44.6 & 49.95 & 25 & - & - & - & 25.87 & 30.6 \\
\hline & Sinus & - & - & - & - & - & - & - & - & - & - & 8.11 & 9.31 \\
\hline & Chest & 3.8 & - & - & - & 3.89 & 5.6 & 6.5 & - & - & - & 5.46 & 4.77 \\
\hline & $\begin{array}{c}\text { Abdomen- } \\
\text { pelvis }\end{array}$ & 4.9 & - & - & - & - & - & - & - & - & - & 7.29 & 8.35 \\
\hline \multirow{4}{*}{$\begin{array}{l}5-10 \\
\text { years }\end{array}$} & Head & 57 & - & - & - & 52.32 & 69.95 & 32.0 & - & - & - & 32.61 & 37.2 \\
\hline & Sinus & - & - & - & - & - & - & - & - & - & - & 8.86 & 9.35 \\
\hline & Chest & 4.8 & - & - & - & 5.26 & 5.65 & 7.6 & - & - & - & 5.59 & 5.4 \\
\hline & $\begin{array}{c}\text { Abdomen- } \\
\text { pelvis }\end{array}$ & 6.1 & - & - & - & - & - & - & - & - & - & 8.82 & 10.25 \\
\hline \multirow{4}{*}{$\begin{array}{l}10-15 \\
\text { years }\end{array}$} & Head & - & - & - & - & 59.2 & 72.28 & 45.0 & - & - & - & 34.78 & 41.1 \\
\hline & Sinus & - & - & - & - & - & - & - & - & - & - & 9.26 & 8.8 \\
\hline & Chest & - & - & - & - & 6.27 & 7.19 & 9.6 & - & - & - & 7.35 & 8.61 \\
\hline & $\begin{array}{c}\text { Abdomen- } \\
\text { pelvis }\end{array}$ & - & - & - & - & - & - & - & - & - & - & 9.22 & 9.3 \\
\hline \multirow{4}{*}{ Adult } & Head & 82 & - & 28.8 & 30.4 & 69.95 & 75.0 & - & - & 50.0 & - & 35.87 & 44.12 \\
\hline & Sinus & - & - & - & - & - & - & - & - & - & - & 11.17 & 9.35 \\
\hline & \begin{tabular}{|l} 
Chest \\
\end{tabular} & 14 & - & 17.7 & 22.3 & 12.39 & 14.0 & - & - & 10.0 & - & 7.78 & 8.6 \\
\hline & $\begin{array}{c}\text { Abdomen- } \\
\text { pelvis }\end{array}$ & 18 & - & 28.6 & 31.0 & 15.59 & 18.0 & - & - & 11.0 & - & 9.94 & 11.05 \\
\hline
\end{tabular}
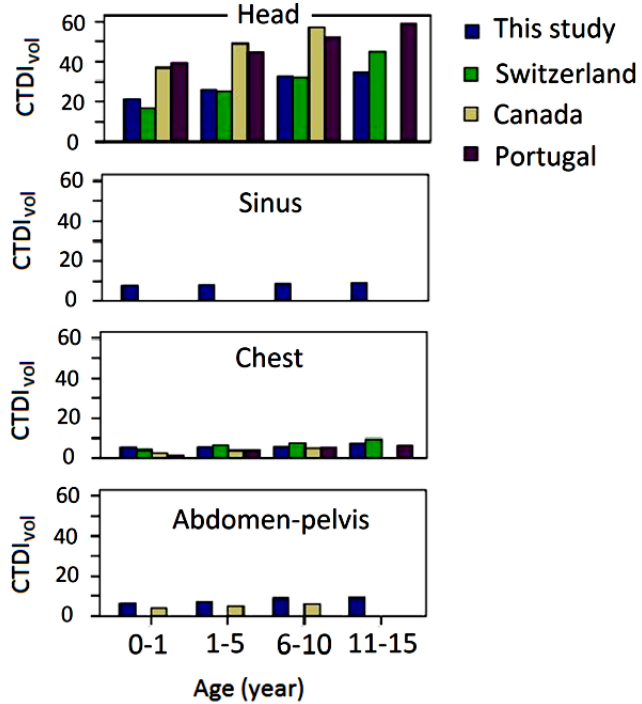

Figure 1. CTDIvol values (mGy) in this study compared with similar studies on pediatric CT examinations in other countries.

The data of the other countries are related to Canada ${ }^{(11)}$, Egypt ${ }^{(25)}$, Portugal ${ }^{(26)}$, Switzerland, and Tehran ${ }^{(19)}$.
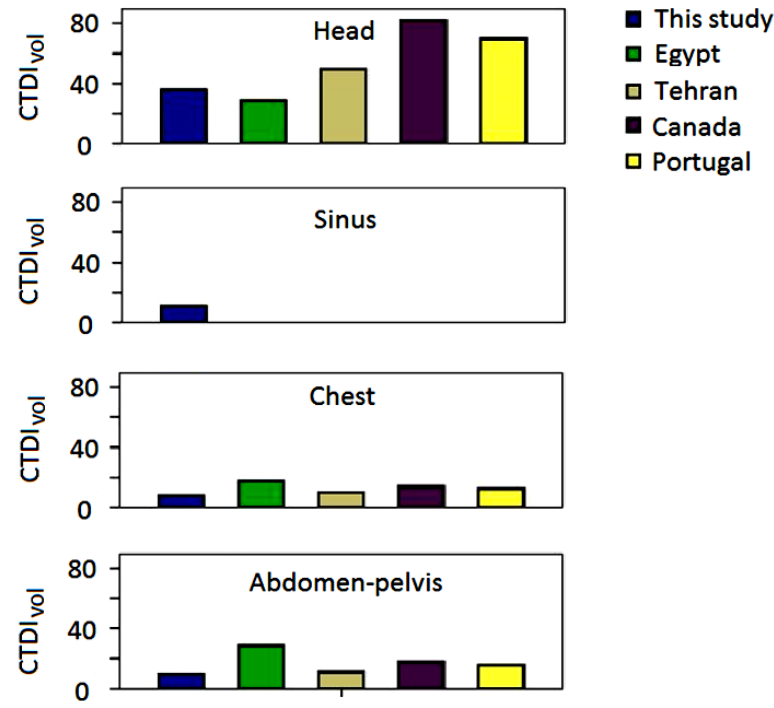

Figure 2. CTDlvol values (mGy) in the present study compared with similar studies on adult CT examinations in other countries. The data of the other countries are related to Canada ${ }^{(11)}$, Egypt ${ }^{(25)}$, Portugal ${ }^{(26)}$, Switzerland, and Tehran ${ }^{(19)}$. 


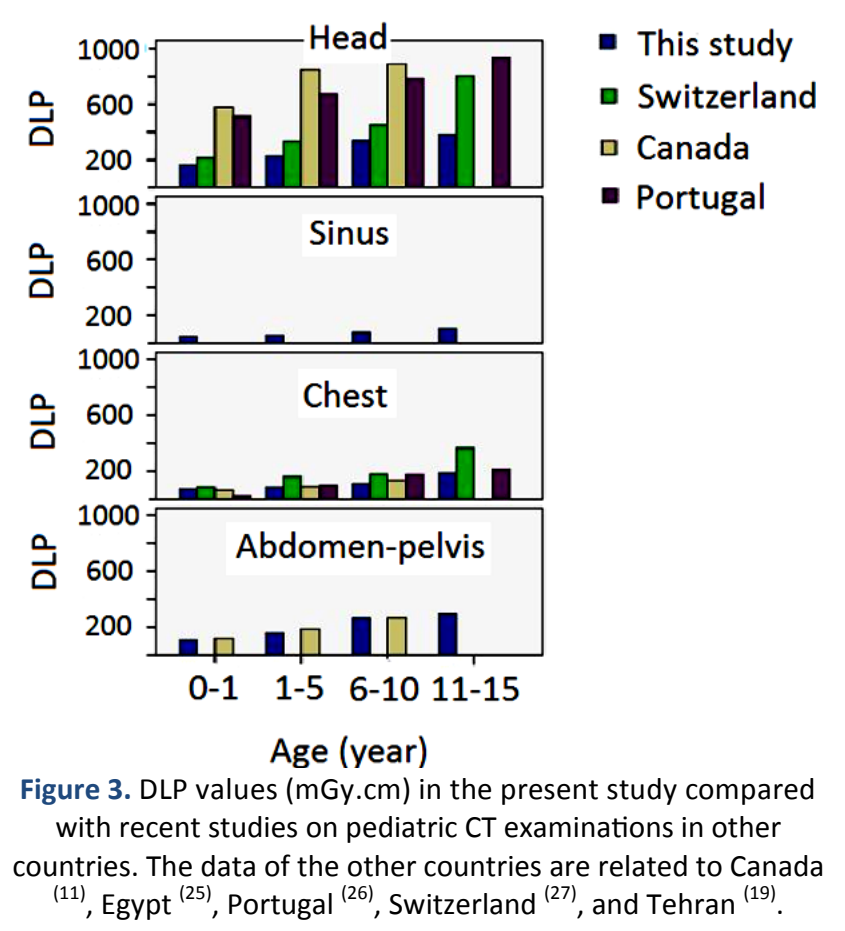

\section{DISCUSSION}

Based on the data in tables 5 and 6, there was an increase in mean values of mean CTDIvol with increasing age of the patients. The reason for this effect can be the fact that the irradiated volume increases with age. There was also an increase in the mean values of DLP with increase in the age of the patients. The mean values of CTDIvol for head CT examinations were higher than the other examinations which were considered in the present study. There was a significant correlation between patient size and CTDIvol for adult patients $(\mathrm{p}=0.02$, Pearson correlation). The lowest and highest variations between maximum and minimum doses were obtained in head and chest protocols, respectively.

The mean values of DLP were also higher for head CT examinations compared to the other examinations. This might be due to more absorption of radiation in the skull. The differences between the CTDIvol and DLP for head and abdomen-pelvis was less than the other cases and this might be due to higher scan length in the abdomen-pelvis examination. Based on CTDIvol and DLP values for selected
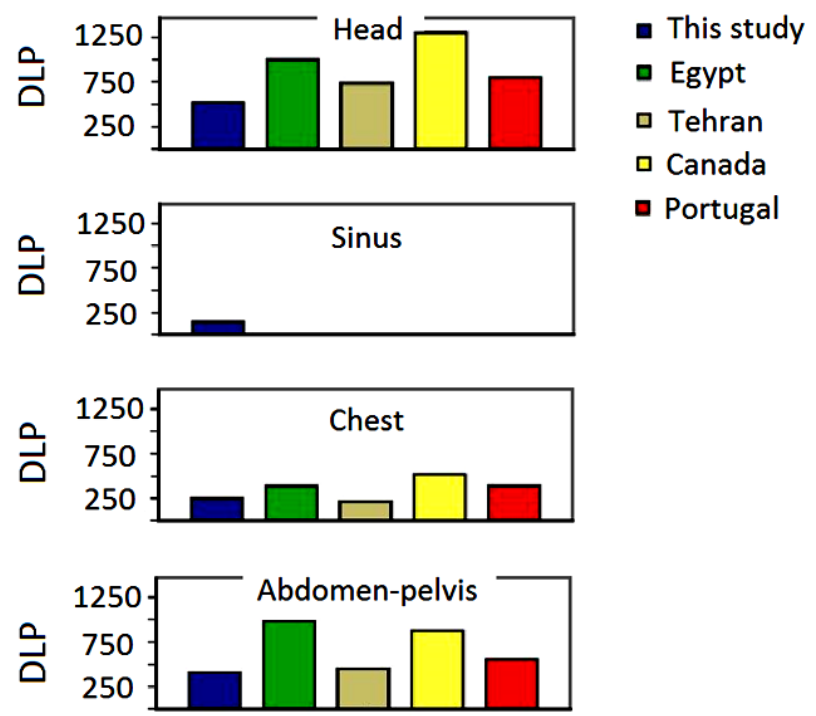

Figure 4. DLP values (mGy.cm) in the present study compared with recent studies on adult CT examinations in other countries. The data of the other countries are related to Canada ${ }^{(11)}$, Egypt ${ }^{(25)}$, Portugal ${ }^{(26)}$, Switzerland ${ }^{(27)}$, and

protocols, CTDIvol and DLP are higher for pediatric (0-1 year) CT scanning of chest and abdomen-pelvis sites in Iran (figure 1). It may be correlated with obesity trends in Iran (28). CTDIvol increased with patient age in children for the chest protocol, but the effective dose is greater for 0-1 year-old patients than 10 year-old ones, which may be due to more sensitivity of younger children. For adult patients, CTDIvol and DLPs are comparable and mainly lower than those reported in same surveys in other countries (figures 1 and 2). This effect represents a good situation in dose reduction methods in Iran. There are differences in doses reported in the present study and a former study by Parsi, et al. in Tehran province (29). Unfortunately, there is no data about the distribution of doses in the study by Parsi, et al. In addition, only dose indicator of CTDIvol was reported in their study and it was insufficient and the DRL was not reported for children.

Two approaches were considered for calculating the ED in the present study. The first approach was the use of pre-tabulated Monte Carlo based on ImpactDose software. The second one was application of conversion factor to calculate the ED separately for different 
irradiated parts of body. By comparing the ED data from the ImpactDose software and from the DLP method (table 7), there was a significant difference between these two data sets ( $p$-value less than 0.01). The ED data from the ImpactDose software were normally higher than the data corresponding data from the DLP method (except for the sinus examination). The differences were also higher for the abdomen-pelvis protocol. The obtained results indicated that the use of DLP method always underestimated the ED, and it will have underestimation in calculation of radiation cancer risk. When there is a need for accurate risk estimation, the ED should be calculated by considering scan range and acquisition parameters. ImpactDose phantom can be modified according to patient effective diameter, age, gender for easily accessibility. Based on the data in table 7, for the ED values in different CT examinations and patients age groups, there was no constant trend for mean ED with increasing the age of the patients. This may be to the higher radiation sensitivity of organs in lower ages, and also increasing of the irradiated volume with age. The mean value of ED was higher for the chest and abdomen-pelvis examinations, compared to the head and sinus examinations. More focus on these data clears that the highest radiation dose was imposed to patients during the abdomen-pelvis protocol and the lowest radiation is for the sinus protocol.

Considering the data of CTDIvol presented in table 8 and in figures 1 and 2 from the present study for Iran and the other countries (Canada, Egypt, Portugal, Switzerland, and Tehran (Iran)), the data for the adult patients are indicating that, except for some data points, the CTDIvol values in Iran was less than the CTDIvol values in the other countries. This can be accounted as an advantage for the examinations in Iran. The difference between the values from various countries may be due to the differences in the types and models of CT equipment, training of the staff, the techniques applied by the staff (for example the length of scanning) and other factors. The mean data of CTDIvol for sinus examination were not reported in the other studies. From the data table 8 , it is apparent that the present study is a more comprehensive evaluation of dose quantities for different CT examination protocols as well as patient age groups. Based on these data it is evident that CTDIvol for head examination is higher than the other examinations (sinus, chest, and abdomen-pelvis) for different countries. The comparison of the CTDIvol data from the present study for the pediatrics groups $(0-1$ year, 1-5, and 6-10, 11-15 years old) with the same age groups from the Canada (11), shows that the CTDIvol in the head examinations in Iran was less than the Canadian groups. The effect is reverse for the chest and abdomen-pelvis pediatric patients from Iran and Canada as well as Portugal. The study by Thakur, et al. lacks the CTDIvol data for the Sinus protocol. In some cases there are large differences between the values for different countries.

This was the first study on patient dose reporting according to age from CT imaging for four protocols of head, sinus, chest and abdomen -pelvis. In comparison with other countries, the calculation of ED by Monte Carlo based software offers a good opportunity for the risk estimation and assessment of risk versus benefit rule.

The survey results can be used as a baseline information for future evaluations of CT procedures in Iran and for optimization of CT practices. To this end, it is possible to find centers which have DRL values higher than the NDRL and apply limitation procedures for them. The high DRLs of these centers may be due to the use of old CT unit, personals' low education or skills, or the use of inadequate procedure. The obtained results would be useful for the Iranian regulatory body in performance of optimization measures.

There are different trends in CTDIvol and ED. It proves that CTDIvol and DLP are not suitable parameters for the presentation of national reference levels (30). They can overestimate or underestimate the risk of radiation. The use of more patients and scan specific quantity of ED is recommended in definition of NDRL, especially in the computed tomography in which the scan and exposure parameters of $\mathrm{kVp}$, scan range and slice thickness can affect the ED. It is suggested 
determining total and organ risks for the Iranian population in CT examinations by using ImpactDose software and biological effects of ionizing radiation (BEIR) VII report (31).

\section{CONCLUSION}

The present study provided a useful and real report of patients' doses from computed tomography of four protocols of head, sinus, chest and abdomen-pelvis for all age groups of children and adults. The data can be used for practical estimation of risk of ionizing radiation in the Iranian population, and can be also used as an exposure guideline or diagnostic reference level to assess exposure parameters and dose optimization in clinical CT scans.

Values of CTDIvol were lower than reported for other countries. However, doses were higher in Iran compared to other studies in terms of DLP. The use of DLP, by involving the scan range, is a better method for calculation of the patient dose. ED is a more useful dose quantity in the assessment of stochastic risks of ionizing radiation, and it is related to patient size, age, gender and an irradiated part of body.

\section{ACKNOWLEDGEMENTS}

The authors are grateful to CT technologists for their cooperation in the present survey as well as the staff of Radiation Protection Department of Atomic Energy Organization of Iran (AEOI) for their kind assistance in the present study.

\section{Conflicts of interest: Declared none.}

\section{REFERENCES}

1. Söderberg M and Gunnarsson M (2010) Automatic exposure control in computed tomography-an evaluation of systems from different manufacturers. Acta Radiol, 51(6): 625-34.

2. Vassileva J and Rehani M (2015) Patient grouping for dose surveys and establishment of diagnostic reference levels in paediatric computed tomography. Radiat Prot Dosimet, 165(1-4): 81-5.

3. Mattsson S and Söderberg M (2011) Radiation dose management in CT, SPECT/CT and PET/CT techniques. Radiat Prot Dosimet, 147(1-2): 13-21.

4. Bebbington $N$, Burniston $M$, Edyvean $S$, Fraser $L$, Julyan $P$, Parkar N, et al. (2016) UK national reference doses for CT scans performed in hybrid imaging studies. J Nucl Med, $\mathbf{5 7}$ (2): 594.

5. Brix G, Nekolla EA, Borowski M, Noßke D (2014) Radiation risk and protection of patients in clinical SPECT/CT. Eur J Nucl Med Mol Imag, 41(1): 125-36.

6. Valentin J (2006) Managing patient dose in multi-detector computed tomography (MDCT). ICRP Publication 102. Ann ICRP, 37(1): 1-79, iii.

7. Protection IR. Safety in Medicine (1996) ICRP Publication, 73: 1-47.

8. Christner JA, Kofler JM, McCollough CH (2010) Estimating effective dose for $\mathrm{CT}$ using dose-length product compared with using organ doses: consequences of adopting International Commission on Radiological Protection Publication 103 or dual-energy scanning. Am J Roentgenol, 194 (4): 881-9.

9. Kalra MK, Maher MM, Toth TL, Hamberg LM, Blake MA, Shepard J-A, et al. (2004) Strategies for CT radiation dose optimization. Radiology, 230(3): 619-28.

10. Shannoun F, Zeeb H, Back C, Blettner M (2006) Medical exposure of the population from diagnostic use of ionizing radiation in Luxembourg between 1994 and 2002. Health Phys, 91(2): 154-62.

11. Thakur $Y$, Bjarnason TA, Baxter $P$, Griffith $M$, Eaton $K$ (2016) Radiation dose survey for common computed tomography exams: 2013 British Columbia results. Canadian Association of Radiologists Journal, 67(1): 88-95.

12. Najafi M, Deevband MR, Ahmadi M, Kardan MR (2015) Establishment of diagnostic reference levels for common multi-detector computed tomography examinations in Iran. Australas Phys Eng Sci Med, 38(4): 603-9.

13. Hart D, Wall B, Hillier M, Shrimpton P (2010) Frequency and collective dose for medical and dental X-ray examinations in the UK, 2008. Health Protection Agency.

14. Mettler Jr FA, Thomadsen BR, Bhargavan M, Gilley DB, Gray JE, Lipoti JA, et al. (2008) Medical radiation exposure in the US in 2006: preliminary results. Health Phys, 95(5): 502-7.

15. Radiation UNSCotEoA (2000) Sources and effects of ionizing radiation. UNSCEAR 2000 report to the General Assembly, with scientific annexes. Volume II: Effects. 2000.

16. Janbabanezhad-Toori A, Deevband M, ShabestaniMonfared A, Abdi R, Nabahati M (2016) Pediatric dose assessment in common CT examination towards establishment of related regional DRL in Mazandaran, Iran. Int J Radiat Res, 14(3): 251-6.

17. Sohrabi M, Parsi M, Tabrizi SH (2019) Statistical analysis for obtaining optimum number of CT scanners in patient dose surveys for determining national diagnostic reference levels. European Radiology, 29(1): 168-75. 
18. Toori AJ, Shabestani-Monfared A, Deevband M, Abdi R, Nabahati M (2015) Dose assessment in computed tomography examination and establishment of local diagnostic reference levels in Mazandaran, Iran. J Biomedical physics \& engineering, 5(4): 177.

19. Sohrabi M, Parsi M, Mianji F (2017) Determination of national diagnostic reference levels in computed tomography examinations of Iran by a new quality control-based dose survey method. Radiat Protect Dosimetry, 179(3): 206-15.

20. Valentin J (2008) The 2007 recommendations of the international commission on radiological protection: Elsevier International Commission on Radiological Protection, 2008.

21. AAPM. Use of Water Equivalent Diameter for Calculating Patient Size

and Size-Specific Dose Estimates (SSDE) in CT. report. 2014. Report No.: 220.

22. Shrimpton P, Hillier M, Lewis M, Dunn M (2005) Doses from computed tomography (CT) examinations in the UK2003 review: NRPB Chilton.

23. Khursheed A, Hillier M, Shrimpton P, Wall B (2002) Influence of patient age on normalized effective doses calculated for CT examinations. The British Journal of Radiology, 75(898): 819-30.

24. Muhogora WE, Ahmed NA, Almosabihi A, Alsuwaidi JS, Beganovic A, Ciraj-Bjelac O, et al. (2008) Patient doses in radiographic examinations in 12 countries in Asia, Africa, and Eastern Europe: initial results from IAEA projects. Am J Roentgenol, 190(6): 1453-61.

25. Salama DH, Vassileva J, Mahdaly G, Shawki M, Salama A, Gilley D, et al. (2017) Establishing national diagnostic reference levels (DRLs) for computed tomography in Egypt. Physica Medica, 39: 16-24.

26. Santos J, Foley S, Paulo G, McEntee MF, Rainford L (2013) The establishment of computed tomography diagnostic reference levels in Portugal. Radiation protection dosimetry, 158(3): 307-17.

27. Verdun FR, Gutierrez D, Vader JP, Aroua A, Alamo-Maestre LT, Bochud F, et al. (2008) CT radiation dose in children: a survey to establish age-based diagnostic reference levels in Switzerland. European Radiology,18(9): 1980-6.

28. Kelishadi R, Haghdoost A-A, Sadeghirad B, Khajehkazemi R (2014) Trend in the prevalence of obesity and overweight among Iranian children and adolescents: a systematic review and meta-analysis. Nutrition, 30(4): 393-400.

29. Parsi M, Sohrabi M, Mianji F, Paydar R (2017) A "qualitycontrol-based correction method" for displayed dose indices on CT scanner consoles in patient dose surveys. Physica Medica, 38: 88-92.

30. Abdollahi H, Shiri I, Salimi Y, Sarebani M, Mehdinia R, Deevband MR, et al. (2016) Radiation dose in cardiac SPECT/CT: An estimation of SSDE and effective dose. Eur J Radiol, 85(12): 2257-61.

31. Hendee WR (1992) Estimation of radiation risks: BEIR V and its significance for medicine. Jama, 268(5): 620-4. 
